\title{
Prevalencia de infección por Treponema pallidum en individuos atendidos en un centro especializado de Medellín, Colombia
}

\author{
Prevalence of Treponema pallidum infection in individuals treated in a specialized \\ medical center in Medellín, Colombia
}

\section{Prevalência de infecção por Treponema pallidum em indivíduos atendidos num centro especializado de Medellín, Colômbia}

\author{
Jaiberth Antonio Cardona-Arias:'1 Luis Felipe Higuita-Gutiérrez;,2 Juan Carlos Cataño-Correa ${ }^{3}$ \\ 1 MSc en Epidemiología, MSc en Economía aplicada. Universidad de Antioquia. jaiberth.cardona@udea.edu.co. ORcID: https://orcid. \\ org/0000-0002-7101-929X \\ 2 MSc en Educación. Universidad de Antioquia, Universidad Cooperativa de Colombia. hgfelipe87@gmail.com. ORcID: https://orcid. \\ org/0000-0003-1361-3124 \\ 3 MSc en VIH. Fundación Antioqueña de infectología, Universidad de Antioquia. kataju@hotmail.com. orcid: https://orcid.org/0000- \\ 0002-0150-5487
}

Recibido:13/08/2020. Aprobado: 28/08/2021. Publicado: 19/01/2022.

Cardona-Arias JA, Higuita-Gutiérrez LF, Cataño-Correa JC. Prevalencia de infección por Treponema pallidum en individuos atendidos en un centro especializado de Medellín, Colombia. Rev. Fac. Nac. Salud Pública. 2022;40(1):e343212. Dor: https:// doi.org/10.17533/udea.rfnsp.e343212

\section{Resumen}

Objetivo: Describir la prevalencia de infección por Treponema pallidum en individuos atendidos en un centro especializado de Medellín, Colombia, 2019. Metodología: Estudio de corte, con 776 sujetos de población general (aparentemente sanosasintomáticos y sin factores de riesgo para sífilis), 126 hombres que tienen sexo con hombres (HSH) y 190 jóvenes vulnerables (estrato socioeconómico bajo, desempleados y sin finalizar educación básica), captados intramural y extramuralmente en espacios de concentración de HSH y organizaciones no gubernamentales. Se empleó fuente de información primaria basada en un encuesta y prueba de detección de anticuerpos IgG, IgM e IgA específicos para T. pallidum, Resultados: La prevalencia de infección por T. pallidum fue $0,53 \%$ en jóvenes vulnerables, 2,19\% en población general y $16,67 \%$ en HSH. La razón de odds de infección en los sujetos con edad entre 51-60 años fue 13,8 (IC $95 \%=3,5-51,5$ ) respecto a la hallada en los jóvenes entre 21-30 años; 12,0 (IC $95 \%=1,8-79,2$ ) en quienes no tienen escolaridad frente a los universitarios; 3,3 (IC 95\% $=1,1-9,9)$ en las personas sin afiliación en salud, comparadas con las del régimen contributivo, y 8,1 (IC $95 \%=2,8-23,0$ ) en quienes tiene relaciones sexuales con grupos clave versus quienes no refieren estas prácticas. Conclusión: Se halló una menor prevalencia de infección por T. pallidum en los jóvenes, seguida de los sujetos de población general y fue mayor en HSH. Se identificaron los grupos de mayor riesgo (entre los tres subgrupos estudiados), que incluyen sujetos excluidos de los programas de prevención, con confluencia de factores de riesgo sexual y privación socioeconómica.

---------Palabras clave: Colombia, factores de riesgo, prevalencia, sífilis, Treponema pallidum. 


\begin{abstract}
Objective: To describe the prevalence of Treponema pallidum infection in individuals treated in a specialized medical center in Medellín, Colombia in 2019. Methodology: A cross-sectional study was conducted, including 776 subjects of the general population (apparently healthy-asymptomatic and without risk factors for syphilis), 126 men who have sex with men (msm) and 190 vulnerable young people (low socioeconomic strata, unemployed and low educational level) which were identified within and beyond spaces of concentration of msm and nongovernmental organizations. A survey and a detection test for IgG, IgM and IgA for T. pallidum specific antibodies were used as the primary information source. Results: Prevalence of T. pallidum infection was $0.53 \%$ in vulnerable young participants, $2.19 \%$ in the general population and $16.67 \%$ in $\mathrm{msm}$. The odds ratio of infection in subjects aged between 51 and 60 years was $13.8(95 \% \mathrm{ci}=3.5-51.5)$ compared to that
\end{abstract}

found in young people aged between 21 and 30 years; 12.0 $(95 \% \mathrm{ci}=1.8-79.2)$ comparing those with low educational level to university students; $3.3(95 \% \mathrm{ci}=1.1-9.9)$ for people without health insurance compared to those belonging to the contributory health insurance regime; and 8.1 (ci $95 \%=2.8$ 23.0) in those who have sex with key groups versus those who do not report these practices. Conclusion: A low prevalence of T. pallidum infection was found in young participants, followed by subjects belonging to the general population and was higher in msm. Groups with the highest risk were identified (among the three subgroups studied), including subjects excluded from prevention programs, with a confluence of sexual risk factors and socioeconomic deprivation.

-----Keywords: Colombia, risk factors, prevalence, syphilis, Treponema pallidum

\section{Resumo}

Objetivo: Descrever a prevalência de infecção por Treponema pallidum em indivíduos atendidos num centro especializado de Medellín, Colômbia, 2019. Metodologia: Estudo de coorte, com 776 pessoas da população em geral (aparentemente saudáveis-assintomáticos e sem fatores de risco para sífilis), 126 homens que fazem sexo com outros homens (HSH) e 190 jovens vulneráveis (classe socioeconômica baixa, desempregados e sem finalizar o ensino fundamental I e II), captados intramural e extramuralmente em espaços de concentração de HSH e organizações não governamentais. Foi usada uma fonte de informação primária baseada numa pesquisa e teste de detecção de anticorpos IgG, IgM e IgA específicos para T. pallidum. Resultados: A prevalência de infecção por T. pallidum foi de $0,53 \%$ em jovens vulneráveis, $2,19 \%$ na população geral e $16,67 \%$ em HSH. A razão de odds ratio de infecção nos indivíduos na faixa etária de 51-60 anos foi 13,8 (IC95\% = 3,5-51,5) em relação a que foi encontrada nos jovens entre 21-30 anos; 12,0 (IC95\% = 1,8-79,2) em quem não tem escolaridade comparada com os universitários; $3,3($ IC95\% $=1,1-9,9)$ nas pessoas sem acesso aos serviços de saúde comparadas com as do regime contributivo, e 8,1 $(\mathrm{IC} 95 \%=2,8-23,0)$ em quem tem relações sexuais com gruposchave vs. quem não tem estas preferências. Conclusão: Foi encontrada uma menor prevalência de infecção por T. pallidum nos jovens seguida dos indivíduos da população geral e foi maior em HSH. Foram identificados os grupos de maior risco (entre os três subgrupos estudados), que incluem sujeitos excluídos dos programas de prevenção, com confluência de fatores de risco sexual e privação socioeconômica.

-Palavras-chave: Colômbia; Fatores de risco; Prevalência; Sífilis; Treponema pallidum.

\section{Introducción}

Según datos de la Organización Mundial de la Salud (oms), cada día se presentan más de un millón de infecciones de transmisión sexual (ITS) en personas con edades entre los 15 y 49 años en el ámbito mundial, con cerca de 376 millones de casos atribuibles a clamidiosis, gonorrea, tricomoniasis y sífilis. Esta última puede transmitirse por contacto con lesiones genitales, anales, del recto, los labios o la boca; también se presenta de madre a hijo en la gestación $[1,2]$.

La sífilis es causada por Treponema pallidum, que genera una infección crónica y sistémica, cuyo espectro clínico incluye las siguientes fases: 1) sífilis primaria o lesión primaria (chancro), con linfadenopatía regional; 2) sífilis secundaria o estado diseminado, con bacteriemia secundaria, adenitis linfática generalizada y lesiones en mucosas y piel; 3) latencia subclínica, y 4) en un tercio de los pacientes sin tratamiento se presenta la sífilis tardía, con lesiones mucocutáneas, osteoarticulares, parenquimatosas, aneurismas y daño del sistema nervioso central.

Para su diagnóstico, se dispone de pruebas no treponémicas para detección de anticuerpos IgG e IgM, prueba rápida para reaginas plasmáticas (Rapid Plasma Reagin, RPR) y laboratorio de investigación de enfermedades venéreas (Venereal Disease Research Laboratory, VDRL), así como pruebas treponémicas de mayor espe- 
cificidad: el método de absorción de los anticuerpos treponémicos fluorescentes (Fluorescent Treponemal Antibody Absorption, FTA-ABS) y la microhemaglutinación frente a T. pallidum (Microhemagglutination Treponema pallidum, MHA-TP). Su tratamiento se basa en una dosis única de bencilpenicilina benzatínica de 2,4 millones de unidades $[3,4]$.

La sífilis registra alrededor de 6 millones de infecciones por año, ocasiona 200 mil mortinatos y muertes neonatales, y 44 mil nacimientos prematuros, constituyéndose en una de las principales causas de muerte en recién nacidos. Esta infección es un grave problema de salud pública, debido a su tendencia al aumento, problemas de suministro mundial de bencilpenicilina benzatínica, pérdida de eficacia de algunos medicamentos por su mal uso, subdiagnóstico en la mayoría de grupos poblacionales, y por generar violencia doméstica y estigmatización $[1,2,5]$.

Debido a la grave situación mundial en este campo, la oms publicó la Estrategia mundial del sector de la salud contra las infecciones de transmisión sexual, 2016 2021, con los siguientes objetivos: disminuir la morbilidad y mortalidad relacionada con esta causa; mejorar la salud sexual de las personas y aumentar su bienestar; acelerar y focalizar iniciativas de prevención; "facilitar el acceso de las personas a la información sobre su estado" de portador; mejorar el acceso a tratamiento y cuidados en salud, y mitigar la estigmatización y la discriminación [6, p. 7].

A pesar de este tipo de estrategias, en Colombia no se dispone de un sistema robusto de vigilancia de la sífilis en población general o en grupos de riesgo (que en Colombia son principalmente los hombres que tienen sexo con hombres (HSH), personas con virus de la inmunodeficiencia humana (VIH), presos y trabajadores sexuales), dado que los esfuerzos giran en torno de las gestantes y sus hijos. En este orden de ideas, se dispone de la "Guía de práctica clínica (GPC) basada en la evidencia para la atención integral de la sífilis gestacional y congénita" [7, p. 1] y el "Protocolo de vigilancia en salud pública de la sífilis gestacional y congénita” [8, p. 1].

En el caso de la sífilis congénita y neonatal en Colombia, se ha destacado la disminución del evento por la unificación de las fichas de notificación y la implementación de la nueva guía de práctica clínica; aunque persisten problemas de "sub-registro y silencio epidemiológico en parte del territorio nacional” [8, p. 4]. Además, algunas cifras evidencian retraso en el cumplimiento de las metas nacionales, dado que se presenta una incidencia de sífilis congénita de 1,1 / 1000 nacidos vivos y sífilis gestacional de 6,6 / 1000 nacidos [8, p. 4], lo que resulta grave por su relación causal con un $80 \%$ de los casos al aborto, muerte fetal, parto pretérmino e infección del recién nacido o sífilis congénita, que puede ir acompañada de diferentes grados de discapacidad e inclusive la muerte [9].

En la producción científica de Colombia, también se observa un énfasis en temas clínicos o en sífilis congénita y neonatal, con pocos estudios de prevalencia en otras poblaciones; por ejemplo, con la estrategia de búsqueda "(ab:(sifilis)) AND in:(“col")", en la base de datos SciELO, se generan cerca de 50 resultados, la mayoría en temas clínicos o sífilis congénita y neonatal, con pocos estudios en población general, HSH $\mathrm{u}$ otro tipo de poblaciones en riesgo. Por su parte, en PubMed $\AA$, las búsquedas “(sifilis[Title/Abstract]) AND prevalence[Title/Abstract]" y "(sifilis[Title/Abstract]) AND MSM[Title/Abstract]" solo generan siete resultados; y con "(sifilis[Title/Abstract]) AND adolescents[Title/ Abstract]" y "(sifilis[Title/Abstract]) AND young[Title/ Abstract]" se obtiene un estudio, ninguno corresponde a población colombiana.

Los pocos estudios disponibles en Colombia son en bancos de sangre; así, en 37044 donantes de Barranquilla, la prevalencia de sífilis fue de $0,93 \%$, con asociación estadística con el sexo, la edad y la ocupación (mayor en hombres, adultos mayores y desempleados) [10], y en 65535 donantes de Medellín fue de 0,6\%, siendo mayor en los hombres [11]. Sin embargo, no se dispone de estudios en grupos de alto riesgo sexual como los HSH y jóvenes en condiciones de vulnerabilidad social y económica; en la búsqueda avanzada en las bases de datos SciELO y PubMed®, solo se halló un estudio, con 569 adolescentes de población general de Medellín, en quienes no se encontraron casos de sífilis [12].

Por lo anterior, se realizó este estudio con el objetivo de describir la prevalencia de infección por $T$. pallidum en individuos atendidos en un centro especializado de Medellín, Colombia, 2019. Es importante precisar que por población general se entienden personas aparentemente sanas-asintomáticas y sin factores de riesgo para sífilis; los jóvenes con vulnerabilidad socioeconómica corresponden a personas menores de 25 años, de estrato socioeconómico bajo (1 y 2), baja cualificación académica (no terminaron sus estudios de básica secundaria) y desempleados. Además, el lugar de estudio, la Fundación Antioqueña de Infectología (FAI), es una institución prestadora de servicios de salud sin ánimo de lucro, que presta atención especializada en patologías relacionadas con enfermedades infecciosas, en tres ejes: atención en salud, educación médica y acciones de promoción de la salud y gestión del riesgo.

La comparación de la prevalencia en los tres grupos, teniendo como referente a las personas de población general, permitió generar evidencia sobre la situación de los jóvenes vulnerables y los HSH, en quienes se presume un mayor riesgo de contraer sífilis y, por consiguiente, necesidad de un abordaje clínico y de salud pública que 
sean acordes con sus realidades, cotidianidad y factores de riesgo singulares.

\section{Metodología}

\section{Tipo y población de estudio}

En este estudio de tipo transversal, se incluyeron 1092 sujetos, 776 de población general, 126 HSH y 190 jóvenes en condiciones de vulnerabilidad socioeconómica, pertenecientes a una organización social. Todos fueron atendidos en la FAI, institución que capta pacientes de manera intramural y extramural en espacios ciudadanos de concentración de HSH (saunas, turcos, salas de reuniones en fundaciones, generalmente ubicados en el centro de la ciudad) y por los miembros de una organización no gubernamental que capta jóvenes vulnerables.

Se trabajó con la totalidad de la población institucional, por lo que no se aplicó tamaño de muestra ni muestreo.

\section{Recolección de la información}

Se utilizó una fuente de información primaria, basada en una encuesta con datos sociodemográficos y riesgos sexuales. Para la detección de la infección por T. pallidum se usó la prueba SD BIOLINE HIV/Syphilis Duo ${ }^{\circledR}$, inmunoensayo cromatográfico de fase sólida, que detecta anticuerpos IgG, IgM e IgA específicos para T. pallidum, mediante tirillas recubiertas con antígenos de captura recombinante $17 \mathrm{kDa}$, y con sensibilidad y especificidad del 99,7\% [13].

El equipo de trabajo de campo de la FAI, conformado por un médico, el líder de proyectos, la coordinadora del trabajo con comunidades y auxiliares de enfermería y de laboratorio clínico aplicaron la encuesta y efectuaron la toma de muestra de sangre periférica, para su procesamiento en un laboratorio clínico de alta complejidad de la ciudad, según las especificaciones del fabricante de la prueba diagnóstica. La prueba permite detectar infección activa; sin embargo, el protocolo de manejo de pacientes incluye la realización de una prueba confirmatoria adicional y complementación con la historia del paciente, para definir la conducta clínica. En caso de confirmarse la sífilis, un infectólogo o el médico general de enlace a infectología lleva a cabo el tratamiento de los casos que lo requieren.

Se controlaron sesgos mediante recolección de datos por personal médico, manual de trabajo de campo con validez de apariencia (un grupo de expertos verificó la aplicabilidad del manual y 10 sujetos del estudio verificaron la aceptabilidad) para el trabajo extramural, campañas de motivación para los participantes, asesoría pre- y posprueba, y aplicación de pruebas diagnósticas con riesgo de falsos positivos (complemento de la espe- cificidad) o negativos (complemento de la sensibilidad) menores que el $1 \%$.

\section{Análisis estadístico}

Todas las variables fueron categóricas (grupo poblacional, lugar de procedencia, sexo, grupo etario, estado civil, escolaridad, afiliación en salud, presencia o ausencia de factores de riesgo sexual y coinfección con otras ITS); por ello se describieron con frecuencias absolutas y relativas.

Los factores de riesgo sexual fueron definidos por la presencia de una o más de las siguientes conductas: nueva pareja sexual en los últimos 6 meses, perforaciones en los últimos 12 meses, uso de alucinógenos en relaciones sexuales, relaciones sexuales con grupo clave (trabajadoras sexuales, habitantes de calle, personas que se inyectan drogas, HSH, transgénero), recibe psicoactivos o dinero a cambio de relaciones sexuales, relaciones sexuales con personas con ITS y privación de la libertad en últimos 12 meses.

Se determinó la prevalencia de infección por $T$. pallidum en la totalidad de la población. Esta se comparó con condiciones sociodemográficas y de factores de riesgo sexual mediante chi cuadrado de Pearson y cuantificación de la fuerza de asociación con razones de odds, con sus intervalos de confianza del $95 \%$. Se elaboró un modelo de regresión logística, mediante el método "introducir", donde las variables independientes fueron seleccionadas con el criterio de Hosmer-Lemeshow $(p<0,25)$ para identificar posibles confusores y seleccionar las potenciales variables explicativas de la prevalencia de infección; en las variables politómicas se crearon dummy (variables indicadoras), tomando como grupo de referencia aquel con menor prevalencia (en estos casos se hizo corrección del valor $p$ con Bonferroni) y la bondad de ajuste se determinó con el estadístico de Hosmer-Lemeshow. Los análisis se consideraron significativos con valores $p$ menores a 0,05 y se realizaron en SPSS 27.0 (IBM ${ }^{\circledR}$ Corp. Released 2017. IBM ${ }^{\circledR}$ SPSS ${ }^{\circledR}$ Statistics for Windows, Version 27.0. Armonk, NY: IBM ${ }^{\circledR}$ Corp., licencia de la Universidad de Antioquia).

\section{Aspectos éticos}

El estudio contó con aval del comité científico y ético de la FAI (Acta 06 del 14 de mayo del 2020), se ajustó a la Resolución 8430 de 1993 del Ministerio de Salud de Colombia, según la cual es un estudio de riesgo mayor al mínimo [14].

Se diligenció consentimiento informado por escrito, y se garantizó confidencialidad y direccionamiento a la institución prestadora de servicios de salud, para garantizar tratamiento de los positivos.

Un miembro de la FAI anonimizó la base de datos que se entregó a los investigadores, asignando un código 
alfanumérico a cada participante, en lugar de los nombres o números de identificación.

\section{Resultados}

En la población de estudio, el 11,5\% fueron HSH y el $17,4 \%$ jóvenes vulnerables; la mayoría eran de Medellín (72,2\%), mujeres (59,9\%), menores de 30 años (66,1\%), solteros $(85,9 \%)$, con estudios de secundaria $(42,2 \%)$, afiliados al régimen contributivo en salud $(58,4 \%)$ y el factor de riesgo sexual más frecuente fue el cambio de pareja en el último semestre (38,9\%) (véase Tabla 1).

Laprevalenciadeinfección por T.pallidum fue 3,57\%. De los 39 casos positivos, $1(0,92 \%)$ tuvo coinfección con virus de la hepatitis C y $16(1,47 \%)$ con VIH. La única variable que no presentó asociación con la infección fue la práctica de perforaciones en los últimos 12 meses ( $p=0,339$ para la prueba chi cuadrado de Pearson); las

Tabla 1. Descripción sociodemográfica y riesgo sexual de la población de estudio.

\begin{tabular}{|c|c|c|c|}
\hline & Variables y sus niveles & $\mathrm{n}$ & $\%$ \\
\hline \multirow{3}{*}{ Población } & General & 776 & 71,1 \\
\hline & $\mathrm{HSH}$ & 126 & 11,5 \\
\hline & Joven vulnerable & 190 & 17,4 \\
\hline \multirow{3}{*}{ Procedencia } & Medellín & 788 & 72,1 \\
\hline & Otros municipios de Antioquia & 206 & 18,9 \\
\hline & Chocó & 98 & 9,0 \\
\hline \multirow{2}{*}{ Sexo } & Mujer & 654 & 59,9 \\
\hline & Hombre & 438 & 40,1 \\
\hline \multirow{6}{*}{$\begin{array}{l}\text { Grupo etario } \\
\text { (años) }\end{array}$} & Adolescentes $(<21)$ & 354 & 32,4 \\
\hline & $21-30$ & 368 & 33,7 \\
\hline & $31-40$ & 141 & 12,9 \\
\hline & $41-50$ & 84 & 7,7 \\
\hline & $51-60$ & 57 & 5,2 \\
\hline & Mayores de 60 & 88 & 8,1 \\
\hline \multirow{2}{*}{ Estado civil } & Casado - Unión libre & 154 & 14,1 \\
\hline & Soltero & 938 & 85,9 \\
\hline \multirow{6}{*}{ Escolaridad } & Ninguna & 134 & 12,3 \\
\hline & Primaria incompleta & 86 & 7,9 \\
\hline & Primaria completa & 87 & 8,0 \\
\hline & Secundaria completa & 461 & 42,2 \\
\hline & Técnico & 186 & 17,0 \\
\hline & Universitario & 138 & 12,6 \\
\hline \multirow{3}{*}{ Afiliación en salud } & Sin afiliación & 64 & 5,9 \\
\hline & Régimen subsidiado & 390 & 35,7 \\
\hline & Régimen contributivo & 638 & 58,4 \\
\hline \multirow{7}{*}{ Factores de riesgo sexual } & Nueva pareja sexual en los últimos 6 meses & 425 & 38,9 \\
\hline & Perforaciones en los últimos 12 meses & 204 & 18,7 \\
\hline & Uso de alucinógenos en relaciones sexuales & 142 & 13,0 \\
\hline & Relaciones sexuales con grupo clave* & 122 & 11,2 \\
\hline & Recibe psicoactivos o dinero a cambio de relaciones sexuales & 44 & 4,0 \\
\hline & Relaciones sexuales con personas con its & 38 & 3,5 \\
\hline & Privación de la libertad en últimos 12 meses & 15 & 1,4 \\
\hline
\end{tabular}

HSH: Hombres que tienen sexo con hombres; ITS: Infecciones de transmisión sexual.

* Trabajadoras sexuales, habitantes de calle, personas que se inyectan drogas, HSH, transgénero. 
demás manifestaron asociación estadística con la prevalencia de sífilis (véase Tabla 2).

La prevalencia de infección por T. pallidum fue estadísticamente mayor en los HSH $(16,67 \%)$, los hombres (6,85\%), las personas con edad entre $51-60$ años $(12,28 \%)$ y mayores de 60 años $(9,09 \%)$, los casados $(7,14 \%)$, personas sin escolaridad $(8,21 \%)$, sin afiliación en salud $(18,75 \%)$ o del régimen subsidiado $(4,10 \%)$, y en quienes presentaron riesgos sexuales $(5,41$ a $17,21 \%)$ (véase Tabla 2).

Los potenciales factores explicativos de la prevalencia de infección por T. pallidum en el grupo de estudio fueron el grupo etario, la escolaridad, el tipo de afiliación en salud y tener relaciones sexuales con grupos clave; en tal sentido, la odds de infección en los mayores de 50 años fue alrededor de 14 veces la hallada en los

Tabla 2. Prevalencias específicas de infección por Treponema pallidum según las características sociodemográficas y factores de riesgo sexual de la población de estudio.

\begin{tabular}{|c|c|c|c|c|}
\hline \multicolumn{3}{|c|}{ Características sociodemográficas } & $\begin{array}{c}\text { Prevalencia } \\
\text { específica \% (n) }\end{array}$ & $\begin{array}{c}\text { Razón de ODDS } \\
\text { (IC } 95 \%)\end{array}$ \\
\hline \multirow{3}{*}{ Población } & General & & $2,19(17)^{b}$ & 1,0 \\
\hline & $\mathrm{HSH}$ & & $16,67(21)$ & $8,93(4,56-17,47)^{* *}$ \\
\hline & Joven vulnerable & & $0,53(1)$ & $0,23(0,03-1,79)$ \\
\hline \multirow{3}{*}{ Procedencia } & Medellín & & $2,66(21)^{b}$ & 1,0 \\
\hline & Otros municipios de Antioquia & & $6,80(14)$ & $2,66(1,33-5,33)^{* *}$ \\
\hline & Chocó & & $4,08(4)$ & $1,55(0,52-4,62)$ \\
\hline \multirow{2}{*}{ Sexo } & Mujer & & $1,38(9)^{b}$ & 1,0 \\
\hline & Hombre & & $6,85(30)$ & $5,27(2,47-11,21)^{* *}$ \\
\hline \multirow{5}{*}{$\begin{array}{l}\text { Grupo etario } \\
\text { (en años) }^{c}\end{array}$} & $21-30$ & & $3,53(13)^{b}$ & 1,0 \\
\hline & $31-40$ & & $6,38(9)$ & $1,86(0,78-4,46)$ \\
\hline & $41-50$ & & $2,38(2)$ & $0,67(0,15-3,01)$ \\
\hline & $51-60$ & & $12,28(7)$ & $3,82(1,46-10,04)^{* *}$ \\
\hline & Mayores de 60 & & $9,09(8)$ & $2,73(1,10-6,81)^{*}$ \\
\hline \multirow{2}{*}{ Estado civil } & Soltero & & $2,99(28)^{b}$ & 1,0 \\
\hline & Casado - Unión libre & & $7,14(11)$ & $2,50(1,22-5,13)^{*}$ \\
\hline \multirow{6}{*}{ Escolaridad } & Ninguna & & $8,21(11)$ & $6,08(1,32-27,98)^{* *}$ \\
\hline & Primaria incompleta & & $3,49(3)$ & $2,46(0,40-15,02)$ \\
\hline & Primaria completa & & $5,75(5)$ & $4,15(1,00-21,86)^{*}$ \\
\hline & Secundaria completa & & $3,69(17)$ & $2,60(0,59-10,41)$ \\
\hline & Técnico & & $0,54(1)$ & $0,37(0,03-4,09)$ \\
\hline & Universitario & & $1,45(2)^{b}$ & 1,0 \\
\hline \multirow{3}{*}{$\begin{array}{l}\text { Afiliación en } \\
\text { salud }\end{array}$} & Sin afiliación & & $18,75(12)$ & $13,15(5,53-31,26)^{* *}$ \\
\hline & Régimen subsidiado & & $4,10(16)$ & $2,44(1,12-5,31)^{*}$ \\
\hline & Régimen contributivo & & $1,72(11)^{b}$ & 1,0 \\
\hline \multirow{12}{*}{$\begin{array}{l}\text { Factores de } \\
\text { riesgo sexual }\end{array}$} & \multirow{2}{*}{ Nueva pareja sexual en los últimos 6 meses } & No & $2,40(16)^{b}$ & 1,0 \\
\hline & & Sí & $5,41(23)$ & $2,41(1,26-4,62)^{\star *}$ \\
\hline & \multirow{2}{*}{ Uso de alucinógenos en relaciones sexuales } & No & $3,16(30)^{b}$ & 1,0 \\
\hline & & Sí & $6,34(9)$ & $2,08(1,00-4,47)^{*}$ \\
\hline & \multirow{2}{*}{ Relaciones sexuales desprotegidas con grupo clave ${ }^{d}$} & No & $1,86(18)^{b}$ & 1,0 \\
\hline & & Sí & $17,21(21)$ & $11,00(5,67-21,32)^{* *}$ \\
\hline & \multirow{2}{*}{ Recibe psicoactivos o dinero a cambio de relaciones sexuales } & No & $3,24(34)^{b}$ & 1,0 \\
\hline & & Sí & $11,36(5)$ & $3,82(1,42-10,31)^{\star *}$ \\
\hline & \multirow{2}{*}{ Relaciones sexuales con personas con ITS } & No & $2,85(30)^{b}$ & 1,0 \\
\hline & & Sí & $23,68(9)$ & $10,59(4,61-24,33)^{* *}$ \\
\hline & \multirow{2}{*}{ Privación de la libertad en últimos 12 meses } & No & $3,44(37)^{b}$ & 1,0 \\
\hline & & Sí & $13,33(2)$ & $4,32(1,00-19,85)^{*}$ \\
\hline
\end{tabular}

HSH: Hombres que tienen sexo con hombres; ITs: Infecciones de transmisión sexual.

${ }^{*} p<0,05 ;{ }^{* *} p<0,01$.

a. El denominador fue el número de sujetos en cada subgrupo ( $\%$ de fila).

b. Grupo de referencia.

c. No hubo casos en el grupo de adolescentes ( $<21$ años).

$d$. Trabajadoras sexuales, habitantes de calle, personas que se inyectan drogas, HSH, transgénero. 
jóvenes entre 21 y 30 años (13,80 en el rango 51-60 y $14,59$ en mayores de 60$) ; 12$ veces mayor en quienes no tienen escolaridad frente a los universitarios; 3,34 veces en las personas sin afiliación en salud respecto a los del régimen contributivo, y 8,09 veces en quienes tienen relaciones sexuales con grupos clave en comparación con los que no refieren estas prácticas (véase Tabla 3). Vale aclarar que la tabla incluye dos modelos de regresión con las mismas variables independientes; su diferencia es que el modelo 2 agrupó algunas categorías de las variables politómicas.

\section{Discusión}

La prevalencia de infección por T. pallidum fue del 0,53\% en jóvenes vulnerables, el 2,19\% en población general y el 16,67 \% en HSH; la odds de infección en los mayo-

Tabla 3. Potenciales factores explicativos de la prevalencia de infección por Treponema pallidum en la población de estudio.

\begin{tabular}{|c|c|c|c|c|}
\hline & & Wald & $p$ & $\begin{array}{c}\text { Razón de ODDS } \\
\text { (IC } 95 \%)\end{array}$ \\
\hline \multirow{16}{*}{$\begin{array}{l}\frac{*}{0} \\
\frac{0}{0} \\
\frac{0}{0} \\
\sum\end{array}$} & Población (HSH / General) & 8,80 & 0,003 & $6,78(1,91-24,02)$ \\
\hline & Grupo etario (años) & 18,32 & 0,001 & \\
\hline & $31-40 / 21-30$ & 5,39 & 0,020 & $3,90(1,23-12,29)$ \\
\hline & $41-50 / 21-30$ & 1,49 & 0,222 & $3,17(0,50-20,26)$ \\
\hline & $51-60 / 21-30$ & 14,12 & 0,000 & $13,80(3,51-54,26)$ \\
\hline & $>60 / 21-30$ & 13,02 & 0,000 & $14,59(3,40-62,55)$ \\
\hline & Escolaridad & 13,04 & 0,023 & \\
\hline & Ninguna / Universitarios & 6,66 & 0,010 & $12,00(1,82-79,24)$ \\
\hline & Primaria incompleta / Universitarios & 0,56 & 0,453 & $2,32(0,26-20,84)$ \\
\hline & Primaria completa / Universitarios & 0,77 & 0,382 & $2,46(0,33-18,40)$ \\
\hline & Secundaria completa / Universitarios & 1,98 & 0,160 & $3,47(0,61-19,59)$ \\
\hline & Técnicos / Universitarios & 0,00 & 0,962 & $1,07(0,07-16,87)$ \\
\hline & Afiliación en salud & 4,77 & 0,042 & \\
\hline & Sin afiliación / Contributivo & 4,75 & 0,029 & $3,34(1,13-9,87)$ \\
\hline & Subsidiado / Contributivo & 0,82 & 0,366 & $1,59(0,58-4,31)$ \\
\hline & Relaciones sexuales con grupo clave* (Sí/No) & 15,35 & 0,000 & $8,09(2,84-23,02)$ \\
\hline \multirow{13}{*}{$\begin{array}{l}\text { * } \\
\frac{0}{0} \\
\frac{0}{0} \\
\frac{0}{z}\end{array}$} & Población (HSH / General) & 9,61 & 0,002 & $6,94(2,04-23,61)$ \\
\hline & Grupo etario (años) & 18,21 & 0,000 & \\
\hline & $31-50 / 21-30$ & 5,46 & 0,019 & $3,73(1,24-11,27)$ \\
\hline & $>50 / 21-30$ & 18,13 & 0,000 & $14,54(4,24-49,86)$ \\
\hline & Escolaridad & 13,10 & 0,011 & \\
\hline & Ninguna / Universitarios & 6,68 & 0,010 & $11,73(1,81-75,86)$ \\
\hline & Primaria / Universitarios & 0,78 & 0,378 & $2,37(0,35-16,05)$ \\
\hline & Secundaria completa / Universitarios & 1,94 & 0,163 & $3,39(0,61-18,90)$ \\
\hline & Técnicos / Universitarios & 0,003 & 0,958 & $1,08(0,07-16,86)$ \\
\hline & Afiliación en salud & 4,83 & 0,090 & \\
\hline & Sin afiliación / Contributivo & 4,81 & 0,028 & $3,34(1,14-9,82)$ \\
\hline & Subsidiado / Contributivo & 0,83 & 0,362 & $1,58(0,59-4,21)$ \\
\hline & Relaciones sexuales con grupo clave** (Sí/No) & 16,18 & 0,000 & $8,16(2,93-22,69)$ \\
\hline
\end{tabular}


res de 50 años fue 14 veces la hallada en los jóvenes entre 21-30 años; 12 veces mayor en quienes no tienen escolaridad respecto a los universitarios; 3,3 veces en las personas sin afiliación en salud comparados con el contributivo, y 8,09 veces en quienes tiene relaciones sexuales con grupos clave versus quienes no refieren estas prácticas. Estos hallazgos muestran la circulación y potencial transmisión del agente en la población de estudio, así como su concentración en grupos desfavorecidos o discriminados en términos sociales y económicos, como lo son los HSH, personas cercanas a la adultez mayor, sin escolaridad y sin afiliación al sistema general de seguridad social en salud, lo que demuestra la relevancia de este tipo de investigaciones.

La prevalencia en el grupo de jóvenes fue baja. Sin embargo, al considerar el hallazgo de cero casos en un estudio previo con adolescentes de Medellín [12], se pone de manifiesto la presencia de prácticas sexuales de riesgo desde edades tempranas en esta población. Esto concuerda con otras publicaciones que refieren inicio temprano de la vida sexual, alto recambio sexual y baja frecuencia del uso de condón en jóvenes $[15,16]$.

Se debe considerar que, pese a su baja ocurrencia, este grupo tiene alto riesgo para otras ITS (especialmente VIH), y algunos autores han reportado un número creciente de casos de sífilis maligna en jóvenes coinfectados con VIH [17].

Además, en otros países se han desarrollado estudios con jóvenes con diferentes tipos de vulnerabilidad, hallando prevalencias más altas que las del actual estudio; por ejemplo, en jóvenes (19-30 años) presos de Chile fue del 4,3\%; y en jóvenes de 15-25 años de la India, del 2,0\% $[18,19]$.

La prevalencia del 2,19\% en población general demuestra una mayor ocurrencia que la obtenida en otros grupos, que si bien son diferentes como los donantes de sangre, comparten dos características relevantes, referidas al ser sujetos aparentemente sanos y sin exposición a factores de riesgo para la infección; este es el caso de un estudio en donantes de sangre de Barranquilla, donde fue del 0,93\% [10], y de Medellín, con el 0,6\%, lo que demuestra heterogeneidad y unos niveles endémicos de la infección por T. pallidum inferiores al $3 \%$ [11].

Pese a las diferencias en la proporción de infectados, esta investigación y las dos citadas coinciden en identificar a los hombres y las personas de mayor edad como los subgrupos de mayor ocurrencia, lo cual da cuenta de cierta consistencia sobre los grupos prioritarios para programas de tamización, prevención y atención.

Adicionalmente, estudios de otros países en población general han reportado prevalencias más bajas. Así, en Guinea Bissau, en 500 personas con edades entre 16 y 49 años, fue del 0,8\% [20]; en 210 mujeres de una universidad de Brasil, del 1,9\% [21], y en 2608 sujetos de una zona rural del suroeste China, fue del 1,2 \% [22].
La prevalencia de infección por T. pallidum en HSH fue $16,67 \%$, lo que evidencia un problema de gran magnitud en este grupo. Pese a que, en la literatura científica nacional, no se dispone de investigaciones comparables, en otros países se cuenta con algunos estudios que muestran una problemática de similar gravedad en varias poblaciones. Así, en 244 sujetos con VIH, se halló una prevalencia de infección por $T$. pallidum del 19,3\%, siendo más afectado el grupo de HSH, en quienes fue del 28,7\% [23]; en 1702 casos de sífilis de un centro diagnóstico de Barcelona, el $93 \%$ correspondió a HSH [24]; otras investigaciones con población institucionalizada demuestran aumento de casos, con mayor afectación de los HSH [25]. En un centro de atención médica analizaron 323 casos de sífilis, de los cuales el $34 \%$ correspondió a HSH [26], $\mathrm{y}$ otros autores indican que cerca de un tercio de $\mathrm{HSH}$ infectados con VIH presentan infección por T. pallidum [27]. Todo ello demuestra que, pese a la escasa evidencia, las publicaciones sobre infección por T. pallidum en HSH se concentran en poblaciones coinfectadas con VIH.

Es de remarcar la novedad de esta investigación, al reportar la prevalencia de sífilis en una población poco estudiada y con alta tasa de infección.

La odds de infección fue más alta en los mayores de 50 años, personas sin escolaridad, sin afiliación en salud y con relaciones sexuales con grupos clave, lo que coincide con otros estudios que refieren la edad como un factor asociado, pero difiere en relación con el reporte de una mayor ocurrencia en los hombres [10,11]. Estas diferencias podrían sustentarse en varios elementos: las variaciones en las poblaciones estudiadas, dado que en Colombia las investigaciones previas se han realizado en donantes de sangre; diferencias en los diseños metodológicos y uso de fuentes secundarias, donde no se incluyen algunas variables sexuales o de afiliación en salud; reporte de asociaciones estadísticas, sin controlar factores de confusión con análisis multivariantes, entre otros factores que deben considerarse en estudios posteriores para permitir la consolidación de evidencia en este campo.

No obstante estas diferencias, esta investigación genera una evidencia sobre el mayor riesgo de sífilis atribuible a las prácticas sexuales y a la pertenencia a grupos desfavorecidos en términos sociales y económicos, como es el caso de los sujetos sin escolaridad ni afiliación en salud. Esto permite aumentar la evidencia sobre la importancia de incorporar mediciones de clase social, para analizar problemáticas de salud [28].

Con respecto a la prueba diagnóstica utilizada y la indicación de tratamiento, la oms recomienda que ante una prueba positiva en entornos de prevalencia baja de sífilis debe administrarse tratamiento a todos los pacientes; mientras que en poblaciones con prevalencia alta de sífilis, como los HSH, un resultado reactivo puede indicar una infección actual o una infección pasada; en estos casos, se debe administrar tratamiento si el paciente refiere no haberlo recibido 
antes. Sin embargo, para diferenciar a personas con infección activa de las que han estado expuestas en el pasado, debe hacerse seguimiento con una prueba de diagnóstico no treponémica, como las RPR o VDRL [29].

En este orden de ideas, un metanálisis de pruebas de diagnóstico rápido halló una sensibilidad combinada del 98,5\% (IC 95\% = 92,1-100\%) y especificidad combinada del 95,9\% (IC $95 \%=81,5-100 \%$ ), destacando las siguientes limitaciones en el estado actual del conocimiento científico en este tipo de pruebas diagnósticas: necesidad de estratificar estos valores según el espectro clínico de la sífilis; desconocimiento en el desempeño diagnóstico en personas inmunocomprometidas o con coinfecciones, y relevancia de mejorar el aseguramiento de la calidad de la prueba, incluyendo entrenamiento del personal, controles de calidad y pruebas de aptitud para la implementación de la prueba [30].

Las limitaciones de esta investigación incluyen las propias de los estudios de corte, es decir, sesgo temporal y ausencia de causalidad en las relaciones estadísticas, así como la ausencia de datos de la historia clínica o información microbiológica adicional que permitiesen clasificar el tipo de sífilis o el espectro clínico de los participantes.

\section{Conclusión}

Se halló una menor prevalencia de infección por T. pallidum en los jóvenes en condiciones de vulnerabilidad socioeconómica, seguida de los sujetos de población general, y fue mayor en HSH.

Se identificó un mayor riesgo de infección (entre los tres subgrupos estudiados) en las personas que tiene relaciones sexuales con grupos de riesgo, personas de mayor edad, sin escolaridad ni afiliación en salud.

Todo ello evidencia un alto riesgo para sujetos generalmente excluidos de los programas de prevención, confluencia de factores de riesgo sexual y privación socioeconómica.

\section{Fuente de financiación}

Grant Gilead - Colombia, Subvención 05239.

\section{Conflicto de intereses}

Ninguno de los autores declara conflicto de intereses para la publicación del manuscrito.

\section{Declaración de responsabilidad}

Se declara que los puntos de vista expresados son responsabilidad de los autores y no de la institución en la que trabajan o de la fuente de financiación.

\section{Declaración de autoría}

Jaiberth Cardona: 1) contribución sustancial a la concepción o diseño del artículo o a la adquisición, análisis o interpretación de los datos 2) participación en el diseño del trabajo de investigación o en la revisión crítica de su contenido intelectual 3) aprobación de la versión final del manuscrito enviado 4) capacidad de responder por las cuestiones relacionadas con la exactitud o integridad de cualquier parte del trabajo

Luis Felipe Higuita: 1) contribución sustancial a la concepción o diseño del artículo o a la adquisición, análisis o interpretación de los datos 2) participación en el diseño del trabajo de investigación o en la revisión crítica de su contenido intelectual 3) aprobación de la versión final del manuscrito enviado 4) capacidad de responder por las cuestiones relacionadas con la exactitud o integridad de cualquier parte del trabajo.

Juan Carlos Cataño: 1) contribución sustancial a la concepción o diseño del artículo o a la adquisición, análisis o interpretación de los datos 2) participación en el diseño del trabajo de investigación o en la revisión crítica de su contenido intelectual 3) aprobación de la versión final del manuscrito enviado 4) capacidad de responder por las cuestiones relacionadas con la exactitud o integridad de cualquier parte del trabajo

\section{Referencias}

1. Organización Mundial de la Salud. Cada día, más de 1 millón de personas contraen una infección de transmisión sexual curable [internet]. 2019 [citado 2020 mar. 20 ]. Disponible en: https:// www.who.int/es/news-room/detail/06-06-2019-more-than-1million-new-curable-sexually-transmitted-infections-every-day

2. Organización Panamericana de la Salud. Nuevas directrices terapéuticas para la clamidiasis, la gonorrea y la sífilis [internet]. 2016 [citado 2020 mar. 20 ]. Disponible en: https://www3.paho.org/hq/ index.php?option $=$ com_content\&view $=$ article\&id $=12446$ : newguidelines-chlamydia-gonorrhoea-syphilis\&Itemid=135\&lang=es

3. Cerrada Bravo T. Sífilis: actualidad, diagnóstico y tratamiento. Rev Fac Med unam. 2003;46(6):236-42.

4. Contreras E, Zuluaga S, Ocampo V. Sífilis: la gran simuladora. Infectio. 2008;12(2):340-7.

5. World Health Organization (wHO). wHо guidelines for the treatment of Treponema pallidum (syphilis). Geneva: wHO; 2016.

6. Organización Mundial de la Salud (oMs). Estrategia mundial del sector de la salud contra las infecciones de transmisión sexual, 2016-2021. Ginebra: oms [internet]. 2016[citado 2020 mar. 20 ]. Disponible en: http://apps.who.int/iris/bitstream/handle/10665/250253/WHO-RHR-16.09-spa.pdf;jsessionid=B5164C C9ECA13B116AAEAC2A2907B9EB? sequence $=1$

7. Colombia, Ministerio de Salud de Colombia, Fondo de Población de las Naciones Unidas (UNFPA). Guía de práctica clínica (GPC) basada en la evidencia para la atención integral de la sífilis gestacional y congénita [internet]; 2014 [citado 2020 mar. 20 ]. Disponible en: https://www.minsalud.gov.co/sites/rid/Lists/ BibliotecaDigital/RIDE/INEC/IETS/gpc $\% 20 \%$ E2\%80\%93guiacompleta-sifilis.pdf 
8. Colombia, Instituto Nacional de Salud. Protocolo de vigilancia en salud pública de la sífilis gestacional y congénita [internet]; 2017 [citado 2020 mar. 20 ]. Disponible en: https://www.ins.gov.co/ buscador-eventos/Lineamientos/PRO\%20Sifilis_.pdf

9. Organización Panamericana de la Salud (OPS). Estrategia y plan de acción para la eliminación de la transmisión materno infantil del VIH y de la sífilis congénita: estrategia de monitoreo regional. Washington DC: OPS; 2014.

10. Martínez-Garcés J, Macías-Vidal M, Maestre-Serrano R, et al. Serorreacción y prevalencia de sífilis en donantes de un banco de sangre de Barranquilla, Colombia. Biomédica. 2019;39(Supl. 1) 63-171. DoI: https://doi.org/10.7705/biomedica.v39i1.4245

11. Patiño Bedoya JA, Cortés Márquez MM, Cardona Arias JA. Seroprevalencia de marcadores de infecciones transmisibles por vía transfusional en banco de sangre de Colombia. Rev. Saúde Pública. 2012;46(6):950-9. DOI: https://doi.org/10.1590/S003489102012000600004

12. Villegas-Castaño A, Tamayo-Acevedo L. Prevalencia de infecciones de transmisión sexual y factores de riesgo para la salud sexual de adolescentes escolarizados, Medellín, Colombia, 2013. Iatreia. 2016;29(1):5-17. DoI: https://doi.org/10.17533/udea.iatreia.v29n1a01

13. Abbott. SD Bioline HIv/Syphilis Duo [internet]. 2018. [citado 2020 abr. 20]. Disponible en: https://www.globalpointofcare.abbott/es/ product-details/sd-bioline-hiv-syphilis-duo.html

14. Colombia, Ministerio de Salud. Resolución 8430, por la cual se establecen las normas científicas, técnicas y administrativas para la investigación en salud (1993 oct. 4).

15. Bouniot-Escobar SV, Muñoz-Vigueras CA, Norambuena-Vergara NR, et al. Prevalencia de conductas sexuales de riesgo en estudiantes de primer año de pregrado de la Universidad San Sebastián, Concepción, Chile, 2016: estudio descriptivo. Rev Colomb Obstet Ginecol. 2017;68(3):176-85. Dor: https://doi.org/10.18597/ rcog. 2799

16. Uribe Rodríguez, Ana Fernanda, Orcasita Pineda, Linda Teresa. Conductas sexuales de riesgo en estudiantes universitarios de la ciudad de Cali-Colombia. Rev. Virtual. Univ. Catol. Norte [internet]. 2009 [citado 2020 mar. 20].;(27):1-31. Disponible en: https://www.redalyc.org/articulo.oa?id=194215432004

17. Fustà-Novell X, Morgado-Carrasco D, Barreiro-Capurro A, et al. Syphilis maligna: A presentation to bear in mind. Actas Dermosifiliogr. 2019;110(3):232-7. Dor: https://doi.org/10.1016/j. ad.2018.02.024

18. Bórquez C, Lobato I, Gazmuri P, et al. Prevalencia del virus de la inmunodeficiencia humana, virus de la hepatitis B y Treponema pallidum en reclusos del Centro de Detención Preventiva de Arica, Chile. Rev Chilena Infectol. 2017;34(5):453-7. Dor: https:// doi.org/10.4067/S0716-10182017000500453
19. Nayyar C, Chander R, et al. Evaluation of risk factors in patients attending STI clinic in a tertiary care hospital in North India. Indian J Sex Transm Dis AIDS. 2015;36(1):48-52. Dor: https://doi. org/10.4103/0253-7184.156715

20. Cowley G, Milne G, Silva E, et al. Prevalence of and risk factors for curable sexually transmitted infections on Bubaque Island, Guinea Bissau. Sex Transm Infect. 2021;97(1):51-55. Dor: https:// doi.org/10.1136/sextrans-2019-054351

21. Suehiro TT, Gimenes F, Souza RP, et al. High molecular prevalence of HPV and other sexually transmitted infections in a population of asymptomatic women who work or study at a Brazilian university. Rev Inst Med Trop Sao Paulo. 2021;(63):e1. DoI: https://doi. org/10.1590/S1678-9946202163001

22. Shi Y, Yang Y, Wang Y, et al. Prevalence and associated factors of Treponema pallidum infection in a rural area of southwestern China. BMC Public Health. 2020;20(1):824. DOI: https://doi. org/10.1186/s12889-020-08952-7

23. Köksal MO, Beka H, Evlice O, et al. Syphilis seroprevalence among HIV-infected males in Istanbul, Turkey. Rev Argent Microbiol. 2020;52(4):266-71. DoI: https://doi.org/10.1016/j. ram.2020.01.002

24. Arando M, Caballero E, Curran A, et al. The epidemiological and clinical characteristics of the epidemic of syphilis in Barcelona. Actas Dermosifiliogr. 2019;110(10):841-9. Dor: https://doi. org/10.1016/j.ad.2019.03.014

25. Fustà X, Fuertes I, Lugo-Colón R, et al. Syphilis epidemics: A descriptive study of patients diagnosed in a tertiary hospital between 2011 and 2015. Med Clin (Barc). 2017;149(12):536-539. DOI: https://doi.org/10.1016/j.medcli.2017.04.024

26. Gállego-Lezáun C, Arrizabalaga Asenjo M, González-Moreno J, et al. Syphilis in men who have sex with men: A warning sign for HIV infection. Actas Dermosifiliogr. 2015;106(9):740-5. DoI: https://doi.org/10.1016/j.ad.2015.05.010

27. González-Domenech CM, Antequera Martín-Portugués I, Clavijo-Frutos E, et al. Syphilis and human immunodeficiency virus infection: an endemic infection in men who have sex with men. Enferm Infecc Microbiol Clin. 2015;33(1):32-6. DoI: https://doi. org/10.1016/j.eimc.2014.07.013

28. Muntaner C, Rocha KB, Borrell C, et al. Clase social y salud en América Latina. Rev Panam Salud Publica. 2012;31(2):166-75.

29. Organización Mundial de la Salud. Nota informativa de la oms sobre el uso de la prueba doble de diagnóstico rápido del VIH y la sífilis [internet]. 2017 [citado 2020 mar. 20 ]. Disponible en: https://www.paho.org/es/documentos/nota-informativa-oms-sobre-uso-prueba-doble-diagnostico-rapido-vih-sifilis-2017

30. Bristow CC, Klausner JD, Tran A. Clinical test performance of a rapid point-of-care syphilis treponemal antibody test: A systematic review and meta-analysis. Clin Infect Dis. 2020;71(Supplement_1):S52-S57. DoI: https://doi.org/10.1093/ cid/ciaa350 